\title{
Potential applications of wastes from energy generation particularly biochar in Malaysia.
}

\begin{abstract}
In Malaysia, abundant agricultural wastes are generated yearly. Therefore it is beneficial to discover new ways to utilize the wastes and employ the carbon source in different industries. Biochar are produced through many heat treatments such as combustion, gasification and pyrolysis for energy generation. The characteristics of these stable carbons such as the physical properties, chemical composition, surface area and surface chemistry determine the effectiveness of the cabon in different applications. Biochar has the ability to retain carbon and this condition is advantageous to prevent the release of carbon back to the atmosphere in the form of carbon dioxide. Application of biochar to soil helps to improve soil fertility and raise agricultural productivity. Biochar also has the ability to reduce carbon dioxide in the flue gas system. There have only been a few studies that discuss on the potential applications of this agriculture waste. The biochar's potential application as carbon sequester for soil application, energy production and dye sorption is being explored in this paper.
\end{abstract}

Keyword: biochar, soil application, energy production 